\title{
Analysis of multiple types of human cells subsequent to bioprinting with electrospraying technology
}

\author{
YU XIN ${ }^{1,2^{*}}$, GANG CHAI $^{1,2^{*}}$, TING ZHANG $^{3 *}$, XIANGSHENG WANG $^{1,2^{*}}$, MIAO QU $^{4}$, ANDY TAN $^{1}$, \\ MELIA BOGARI ${ }^{1}$, MING ZHU ${ }^{1}$, LI LIN ${ }^{1}$, QINGXI HU ${ }^{3}$, YUANYUAN LIU $^{3}$ and YAN ZHANG ${ }^{1,2}$ \\ ${ }^{1}$ Department of Plastic and Reconstructive Surgery, Shanghai 9th People's Hospital; ${ }^{2}$ Shanghai Tissue Engineering Key \\ Laboratory, Shanghai Jiao Tong University School of Medicine, Shanghai 200011; ${ }^{3}$ Rapid Manufacturing Engineering \\ Center, School of Mechatronic Engineering and Automation, Shanghai University, Shanghai 200444, P.R. China; \\ ${ }^{4}$ Clinic for Plastic, Hand and Burns Surgery, RWTH Aachen University Hospital, D-52074 Aachen, Germany
}

Received August 22, 2016; Accepted October 4, 2016

DOI: $10.3892 /$ br.2016.790

\begin{abstract}
The aim of the present study was to investigate bioprinting with electrospraying technology using multiple types of human cell suspensions as bio-ink, in order to lay the initial foundations for the application of the bioprinting technology in tissue engineering. In the current study, six types of human cells were selected and cultured, including human fibroblasts, human adipose-derived stem cells (hADSCs), human periodontal ligament cells (HPDLCs), adult human retinal pigment epithelial cells (ARPE-19), human umbilical vascular endothelial cells (HUVECs) and human gastric epithelial cell line (GES-1). Each cell type was divided into two groups, the experimental and control group. All the experimental group cells were electrosprayed using an electrospraying printer (voltage, $15 \mathrm{kV}$; flow rate, $150 \mu \mathrm{l} / \mathrm{min}$ ) and collected in a petri dish placed $15 \mathrm{~cm}$ away from the needle (needle diameter, $0.5 \mathrm{~mm}$ ). Subsequently, cell viability was detected by flow cytometry with a Live/Dead Viability kit. In addition, the cell morphological characteristics were observed with a phase-contrast microscope after $6 \mathrm{~h}$ of culturing in order to obtain adherent cells, while cell proliferation was analyzed
\end{abstract}

Correspondence to: Professor Yuanyuan Liu, Rapid Manufacturing Engineering Center, School of Mechatronic Engineering and Automation, Shanghai University, 99 Shang Da Road, Shanghai 200444, P.R. China

E-mail: yuanyuan_liu@shu.edu.cn

Dr Yan Zhang, Department of Plastic and Reconstructive Surgery, Shanghai 9th People's Hospital, Shanghai Jiao Tong University School of Medicine, 639 Zhi Zao Ju Road, Shanghai 200011, P.R. China

E-mail: 13651817522@163.com

${ }^{*}$ Contributed equally

Key words: bioprinting, electrospraying technology, human cells, multicellular analysis, tissue engineering using a Cell Counting Kit- 8 assay. The control groups, without printing, were subjected to the same procedures as the experimental groups. The results of the cell viability and proliferation assays indicated a statistically significant difference after printing between the experiments and control groups only for the hADSCs $(\mathrm{P}<0.05)$; by contrast, no significant difference was observed in cell viability and proliferation for the other five cell types $(\mathrm{P}>0.05)$. In addition, there were no observable differences between all experimental and the control groups at any examined time point in the terms of cell morphological characteristics. In conclusion, bioprinting based on electrospraying technology demonstrated no distinct negative effect on cell vitality, proliferation and morphology in the present study, and thus the application of this novel technology to cell printing may provide a promising method in tissue engineering.

\section{Introduction}

Tissue engineering has emerged as an interdisciplinary field that seeks to restore, maintain or improve organ function in vivo using artificial tissue or organs, and is a promising approach for addressing transplantation organ shortage $(1,2)$. The general model of traditional tissue engineering is based on the isolated cell and subsequent cell seeding with exogenous scaffolds (3), in order to obtain the matured tissue substitutes (4). However, the precise placement of a large quantity of multicellular biomaterials in spatial and sequential rapid prototyping remains a limitation of traditional tissue engineering methods. Organs consist of extracellular matrix and different cell types that require specific spatial organization (5). In order to create a complex artificial biosystem, a subtly arranged spatial mold should be constructed, in which different cells, nutrients and biofactors are precisely located to mimic the microenvironment in vivo and to obtain the appropriate biological function (6). Another significant hurdle is providing metabolically appropriate conditions inside of a three-dimensional (3D) tissue construct with limited thickness, which may limit the support for the metabolic demands of engineered cells, particularly for certain metabolically active cells, including cardiomyocytes and hepatocytes (7). 
In addition, vascularization remains another big challenge for maintaining the biological activity of engineered constructs. Out-branching blood vessels usually require numerous days for vascularizing the implanted tissue, while seeded cells are unable to obtain sufficient nutrient support before they consume all the available oxygen within a few hours (8).

Bioprinting, based on layer by layer deposition of cells and/or cell aggregates into a thermo-reversible gel, is defined as a new computer-aided 3D rapid prototyping technology with sequential maturation of the printed structures into a living tissue or organ (9). As a breakthrough in regenerative medicine, this emerging technology is currently adapted to produce a variety of tissue architectures and biomaterials, providing a novel cell-based therapeutic approach (7) for organ loss and failure, which is economical and efficient $(3,10,11)$. For instance, myocardial patches have been successfully formed through the post-printing fusion (12), and fully biological scaffold-free vascular tubular grafts have also been constructed using this technology (4).

Although bioprinting is still a new technique compared with existing traditional tissue engineering methods, this novel approach has various advantages: i) With high-efficiency, the tissue engineering project is simplified and can be performed automatically $(5,13)$; ii) a subtle spatial control of the cell types, extracellular matrix, blends of polymers and other cell inductive particles in well-defined 3D microenvironment can be achieved with computer-aided design (CAD) software (11); iii) bioprinting can be applied in the scalable generation of high-throughput cells $(5,13)$; iv) vascularization of complex constructs can be resolved (3); v) this method offers an effient approach to realize the goal of repair and reconstruction in situ; vi) the production cycle can be significantly shortened and the process has higher repeatability than other techniques; and vii) this method excels at producing a variety of tissue architectures with high physical complexity (10).

Currently, widely used routes are available in bioprinting, ranging from non-jet-based approaches, such as laser-directed writing (LGDW) and photolithography, to jet-based methods (14). LGDW is a micro-patterning technique that can facilitate studies at the single cell level. It utilizes a focused beam to confine and guide cells by exploiting the differences in the refractive indexes of the cells and cell media, and then depositing them onto non-absorbing surfaces (8). However, limited throughput and high complexity have restricted the potential application of LGDW in tissue construction (15). Besides, exposure of excessive thermal energy via laser light onto the cell biolayer and overheating of the cell can be a potential challenges that must be overcome (13). Jet-based technologies are the most commonly used in bioprinting, and are promising for handling various materials, including molecular polymers and micro/nanosized compounds. There are numerous printing techniques based on jet-based technology, such as inkjet printing, electrospraying and electrospinning.

Inkjet printing, based on ink drop ejection (5), was the first method to be used for printing 3D architectures. During $3 \mathrm{D}$ inkjet printing, the ink containing cells, culture medium and gel precursor solution is expelled by the jet pens with specific drop volume, and the energy supplied to the ink, from heating by a setting pulse, is transformed into kinetic energy of drops and heating of the drops. Subsequently, the bio-ink is printed layer-by-layer onto the platform, resulting in 3D structures (5). However, nozzle blockage remains a problem with this process (14), while limitations in spatial resolution and control of the droplet size result in coarse architectures (13). Inkjet printers based on piezoelectric technology have numerous applications in ceramics and polymer printing, and are also used in bioprinting. However, due to problems with ink leakage and formation of mist, more viscous ink is required, thus requiring more power and higher vibration frequency that may cause damage to the cells (15).

Electrospraying generates droplets between the jetting needles, driven by electric fields created by the applied potential difference between the jetting needle and a ground electrode (16). This technique has recently been developed to process living cellular organisms (17), and previous studies have elucidated the flexibility of using electrospraying technology in a range of applications, including tissue engineering and regenerative medicine with cell-based and molecular-based therapies $(18,19)$. Under the voltage driving effect, the liquid is separated into microdroplets and spayed onto a predesigned area. For instance, cell transmission based on electrospraying principles, which is known as 'bio-electrospraying' $(14,18)$, has being increasingly explored. Bio-electrospraying directly uses cell suspensions as ejection materials that form droplets at the microscale to nanoscale in an electric field. This technique can produce single-cell scattering and direct transmission. Thus, electrospraying is regarded as a novel route to analyze cell biochemical characteristics and perform $3 \mathrm{D}$ printing in tissue engineering.

In the present study, the ability to directly electrospray multiple types of human cell suspensions in vivo was demonstrated. In addition, the study is the first to verify that different human cells can maintain high cell viability subsequent to electrospraying under the same set-up conditions. This study demonstrates that electrospraying is a promising technology for building human tissue substitutes in organ loss and failure.

\section{Materials and methods}

Cell harvest and culture. All of the experimental procedures were approved by the Ethics Committee of Shanghai 9th People's Hospital (Shanghai, China). Informed consent was obtained from each patient prior to participation in the study. Six different human cell types were used for this cell printing experiment. Skin fibroblasts were isolated from discarded prepuce tissue of 3 male patients (one aged 6 years and two aged 8 years), who underwent circumcision surgery. The tissue was obtained following surgery and washed immediately with saline solution containing gentamicin. Next, the tissue was washed with antibiotic/antimycotic solution followed by phosphate-buffered saline (PBS; Sigma-Aldrich, St. Louis, MO, USA). The tissue was then cut into $1 \mathrm{~mm}$ sections and placed into a culture dish with Gibco high glucose-Dulbecco's modified Eagle's medium (H-DMEM; Thermo Fisher Scientific, Inc., Waltham, MA, USA) containing Gibco $10 \%$ fetal bovine serum (FBS; Thermo Fisher Scientific, 
Inc.) and $1 \%$ antibiotic/antimycotic (Gibco; Thermo Fisher Scientific, Inc.) solution.

Human adipose-derived stem cells (hADSCs) were isolated from female patients (ages, 36, 42 and 45 years) who underwent abdominal liposuction surgery, and these ADSCs were maintained in Gibco low glucose-DMEM (L-DMEM; Thermo Fisher Scientific, Inc.) containing 10\% FBS and $1 \%$ antibiotic/antimycotic solution. Human periodontal ligament cells (HPDLCs) were also derived from the periodontal ligament of human third molars extracted from 3 healthy donors (14-year-old male; 16-year-old female; 15-year-old female) for orthodontic reasons, who had no clinical signs of chronic periodontal disease. The isolated cells were incubated with high H-DMEM containing $10 \%$ FBS and $1 \%$ antibiotic/antimycotic solution. The abovementioned human tissue separated cells were all obtained from patients undergoing surgery at the Shanghai 9th People's Hospital. Up to three passages were used for the experiments.

In addition, the adult human retinal pigment epithelial (ARPE-19) cell line obtained from American Type Culture Collection (ATCC; Manassas, VA, USA) was used in the present study (passages 5-10), and cells were cultured in DMEM supplemented with $10 \%$ FBS and $1 \%$ antibiotic/antimycotic solution. Furthermore, human umbilical vascular endothelial cells (HUVECs) were purchased from ATCC (Manassas, VA, USA) and maintained in F-12 K (LGC Standards, Barcelona, Spain) supplemented with 10\% FBS, heparin and endothelial cell growth supplement. GES-1, an immortalized human gastric epithelial cell line, was also purchased from ATCC, and cells (passages 5-10) were maintained in Gibco RPMI-1640 (Thermo Fisher Scientific, Inc.) medium supplemented with $10 \%$ FBS, 2 mM L-glutamine (Gibco; Thermo Fisher Scientific, Inc.), $100 \mathrm{U} / \mathrm{ml}$ penicillin and $100 \mathrm{mg} / \mathrm{ml}$ streptomycin.

All the cells were incubated in a $37^{\circ} \mathrm{C}, 5 \% \mathrm{CO}_{2}$, and $99 \%$ relative humidity chamber. The cells were allowed to reach $80 \%$ confluence prior to passaging. The culture medium was replenished with fresh medium every 2 or 3 days.

Cell preparation and cell print suspension. When the cell reached $80 \%$ confluence, they were detached with $0.25 \%$ trypsin-EDTA (Sigma-Aldrich) and then the cell pellets were collected and centrifuged at $524 \mathrm{x} \mathrm{g}$ for $5 \mathrm{~min}$. Following aspiration of the supernatant, the cell pellets were resuspended and each single cell suspension was diluted at a density of $1 \times 10^{6} / \mathrm{ml}$ in the culture medium to obtain the cell print suspension. The experimental and control groups were treated under the same conditions, although there was no printing in the control group. Prior to printing, the printer was prepared within $1 \mathrm{~h}$ in sterile conditions.

Cell printing and cell culture. The cell suspension was resuspended at a final concentration of $10^{6}$ cells $/ \mathrm{ml}$ and shaken vigorously prior to printing. Next, approximately $1-2 \mathrm{ml}$ of the cell suspensions was sequentially conveyed to the cell electrospraying printer each time with the parameters set as follows: Voltage, $15 \mathrm{kV}$; flow rate, $150 \mu \mathrm{l} / \mathrm{min}$. The collecting Petri dish was placed $15 \mathrm{~cm}$ away from the sprayer nozzle (nozzle diameter, $0.5 \mathrm{~mm}$ ). The suspension of each cell group was electrosprayed directly onto the petri dish for $30 \mathrm{~min}$ under sterile conditions and the cells were collected in the culture medium similarly to the culture stage mentioned previously. Subsequent to printing, the yielding cells were centrifuged at $524 \mathrm{x} \mathrm{g}$ for $5 \mathrm{~min}$ and resuspended with culture medium. The medium was changed every 1-2 days duing the 5-day culture. The control groups were synchronously placed under the same sterile conditions as the experimental groups, without printing, they were subsequently treated the same as the experimental group.

Cell viability assay. Following the identification of the required parameters for the generation of printed cells under stable electrospraying conditions (voltage, $15 \mathrm{kV}$; flow rate, $150 \mu \mathrm{l} / \mathrm{min}$ ), the present study attempted to determine whether the electrospraying conditions affected the electrosprayed cells. The changes of cells were evaluated using the cell viability assay. The LIVE/DEAD Viability/Cytotoxicity Assay kit (Thermo Fisher Scientific, Inc.,) was used to analyzed cell viability with two fluorescent agents: Calcein acetoxymethyl (AM), which is retained within live cells and produces an intense uniform green fluorescence (ex/em 495/ 515 nm); and ethidium homodimer-1 (EthD-1), which enters cells with damaged membranes and produces a bright red fluorescence in dead cells (ex/em $\sim 495 / \sim 635 \mathrm{~nm}$ ). First, $50 \mu \mathrm{M}$ working solution of calcein AM was obtained by forming an 80-fold dilution of calcein AM in DMSO. In addition, single cell suspensions at a density of $0.1-5 \times 10^{6}$ cells/Ml were prepared. Subsequently, $2 \mu 1$ calcein AM working solution and $4 \mu \mathrm{l}$ EthD-1 stock solution ( $2 \mathrm{mM}$ ) were added to each milliliter of cells, and the sample was mixed. The cells were then incubated for 15-20 min at room temperature, in the dark. Following the incubation, the samples were analyzed by flow cytometry at an excitation wavelength of $488 \mathrm{~nm}$, measuring green fluorescence emission for calcein AM and red fluorescence emission for EthD-1. The cell population was thus separated into two groups: Live cells showing green fluorescence and dead cells showing red fluorescence.

Morphological characteristic assay. Subsequent to printing, the cells were at a resuspended and diluted single cell suspension at a density of $0.5 \times 10^{6} / \mathrm{ml}$ in the culture medium, and then regularly cultured for 5 days at $37^{\circ} \mathrm{C}$ in a humidified atmosphere containing $5 \% \mathrm{CO}_{2}$ with the media changed twice a week. After $6 \mathrm{~h}$ of incubation, the cells were allowed to adhere to the bottom of the dishes. Cell morphological characteristics were then observed under a phase-contrast microscope between the samples at $6 \mathrm{~h}$ of incubation and up to 5 days of culturing. Within this period, experimental and control groups were treated with same resuspension and culture procedures.

Cell proliferation assay. A total of $100 \mu \mathrm{l}$ cell suspensions (1,000 cells/well) of all types of cells were dispensed in a 96-well plate, and the plate was incubated as mentioned above for a scheduled time period (for 0-5 days). In order to determine the cell proliferation, the cells were washed with PBS and incubated with the Cell Counting Kit-8 (CCK-8; Dojindo Molecular Technologies, Inc., Rockville, MD, USA) along with the culture media at a ratio of 1:10. Subsequently, the plates were incubated for 2-4 h [different incubation times were set to ensure the optical density (OD) values were within 
a precise range $(<3)$ and the incubation time between the control and experimental group of the same cell type was the same. The fibroblast and HPDLC groups were incubated for $2.5 \mathrm{~h}$; hADSCs groups were incubated for $4 \mathrm{~h}$; the ARPE-19, HUVECs and GES-1 groups were incubated for $2 \mathrm{~h}$ ] in an incubator at $37^{\circ} \mathrm{C}$ in a humidified atmosphere containing $5 \% \mathrm{CO}_{2}$. The absorbance in terms of the OD was then read at $450 \mathrm{~nm}$ using a plate reader. The OD values measured the quantity of formazon dye, a product of CCK-8, and cell dehydrogenase, which was proportional to the number of living cells. Thus, the cell proliferation trend was revealed by the OD values at different time-points.

Statistical analysis. All statistical analyses were performed using the GraphPad Prism 6 software (GraphPad Software, Inc., La Jolla, CA, USA). The cell survival rate data are presented as the mean \pm standard deviation $(\mathrm{n}=3)$. The CCK-8 assay data are expressed as the mean \pm standard error of the mean ( $\mathrm{n}=9$ in each group). Statistical analyses were performed using a two-tailed Student's t-test in the CCK- 8 assay between the control group and experimental group of each type of cell, with $\mathrm{P}<0.05$ considered to indicate a statistically significant difference. Each experiment was performed in triplicate.

\section{Results}

Cell viability. Flow cytometry assay results of each cell type in each cell type sample are presented in Fig. 1 and Table I. With the exception of the hADSCs, the mean survival rate of each type, including the fibroblasts, HPDLCs, ARPE-19, HUVECs and GES-1 reached $>90 \%$, and there was no statistically significant difference detected between the experimental and control groups $(\mathrm{P}=0.1362$ in the fibroblast groups; $\mathrm{P}=0.0698$ in the HPDLC group; $\mathrm{P}=0.0664$ in the ARPE-19 group; $\mathrm{P}=0.1217$ in the HUVEC group; $\mathrm{P}=0.0869$ in the GES-1 group). By contrast, evident differences among the three samples of hADSCs were observed, and a statistically significant difference was detected between the printed and unprinted groups $(\mathrm{P}=0.0112)$. According to the results, it was concluded that all experimental groups attained viability $>80 \%$, while other groups excluding the hADSCs achieved a viability of $>90 \%$. Although, there was significant difference in the hADSC group, the difference in the mean survival rate between the printed and unprinted groups was within $5 \%(\mathrm{P}=83.20 \pm 5.10$ in the printed group; $\mathrm{P}=87.57 \pm 5.29$ in the unprinted group). Thus the results indicate that the electrospraying process scarcely affected the cell viability.

Morphological characteristic assay. Fig. 2 shows the results of the observation of cellular morphology under a phase-contrast microscope for 5 days after electrospraying. All the cells in the printed groups presented similar growth to the control cells. In addition, there was no observable difference in the morphology and cell density between the printed and unprinted cells at any of the time-points investigated.

Cell proliferation after printing. The CCK-8 assay was used to detect OD values of the experimental and control groups between 0 and 5 days of incubation. As shown in Fig. 3, for all cell types, the cells proliferated stably during the 5-day
A
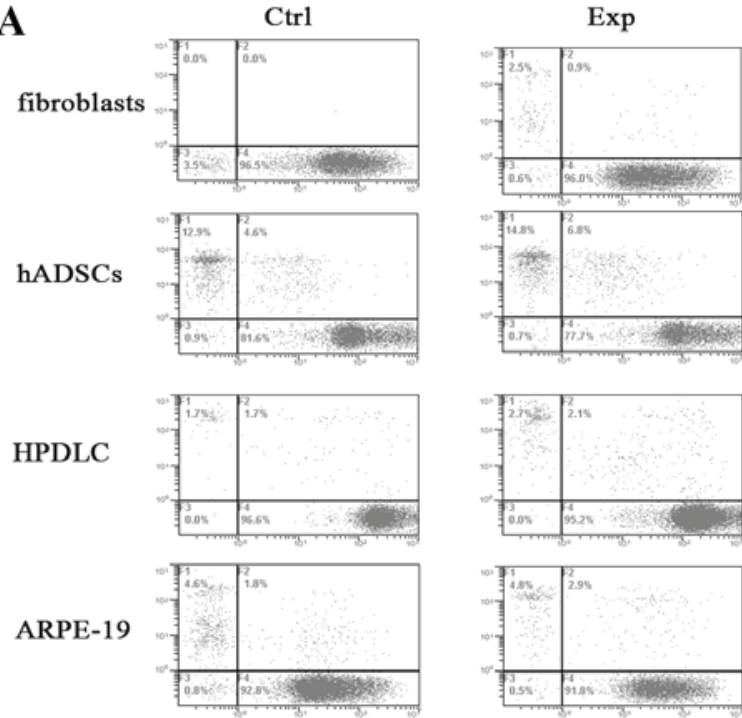

HPDLC
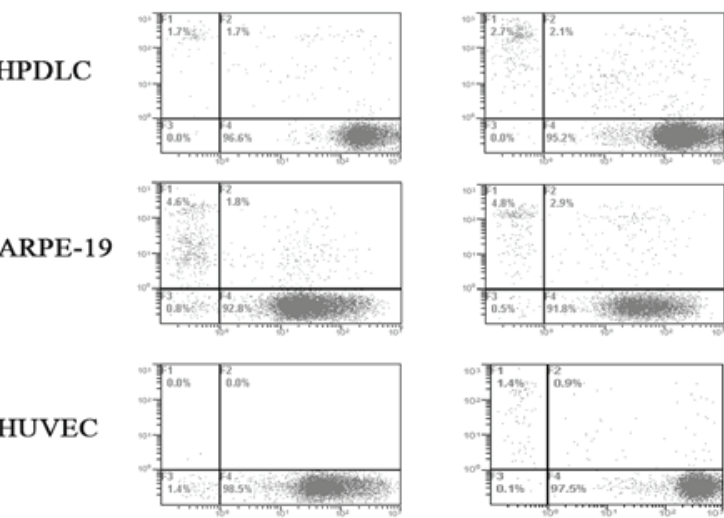

ARPE-19

GES-1
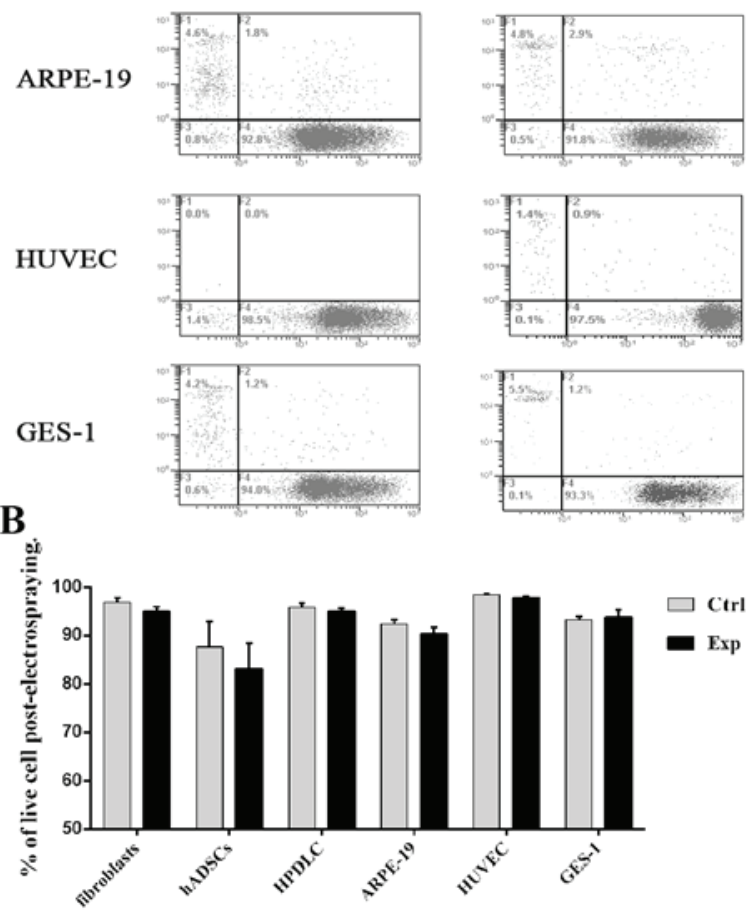

Figure 1. Flow cytometry analysis of cell viability post-electrospraying (A) Live cells appear in the lower right area and dead cells appear in the upper left area of the graphs. (B) The percentage of live cells post-electrospraying in experimental groups was similarly high as that in the control groups (mean \pm standard deviation; $\mathrm{n}=3$ ). Exp, experimental group; $\mathrm{Ctrl}$, control group; hADSCs, human adipose-derived stem cells; HPDLCs, human periodontal ligament cells; ARPE-19, adult retinal pigment epithelial cells; HUVECs, human umbilical vascular endothelial cells; GES-1, human gastric epithelial cells.

culture period, and the cell growth trend was similar between the experimental and control groups. As shown in Table II, the results indicated that there was no significant difference in the OD values between the experimental and control groups for all cells $(\mathrm{P}>0.05)$, with the exception of the hADSCs $(\mathrm{P}=0.0155)$. This suggests that the proliferation rate in the logarithmic growth period and plateau period of the experimental groups was basically consistent with that observed in the control group. Therefore, the printing process did not have a significantly negative effect on the proliferation of the majority of cell types.

As mentioned earlier, all these preliminary results suggest that various types of human cells can be successfully printed by the electrospraying technology. Furthermore, electrosprayed 
Table I. Cytometry assay results of cell survival rate following bioprinting of different cell types.

\begin{tabular}{|c|c|c|c|c|c|c|c|c|c|}
\hline \multirow[b]{3}{*}{ Cell type } & \multicolumn{8}{|c|}{ Survival rate $(\%)$} & \multirow[b]{3}{*}{ P-value } \\
\hline & \multicolumn{2}{|c|}{ Sample 1} & \multicolumn{2}{|c|}{ Sample 2} & \multicolumn{2}{|c|}{ Sample 3} & \multicolumn{2}{|c|}{ Mean survival } & \\
\hline & Exp & Ctrl & Exp & Ctrl & Exp & Ctrl & Exp & Ctrl & \\
\hline Fibroblasts & 96.0 & 96.5 & 94.7 & 98.0 & 94.1 & 96.2 & $94.93 \pm 0.97$ & $96.90 \pm 0.96$ & 0.1362 \\
\hline hADSCs & 77.7 & 81.6 & 91.3 & 91.7 & 88.4 & 90.3 & $83.20 \pm 5.10$ & $87.57 \pm 5.29$ & 0.0112 \\
\hline HPDLCs & 95.2 & 96.6 & 94.1 & 95.0 & 95.5 & 96.0 & $94.93 \pm 0.74$ & $95.87 \pm 0.81$ & 0.0698 \\
\hline ARPE-19 & 91.8 & 92.8 & 89.2 & 91.5 & 90.0 & 92.9 & $90.33 \pm 1.33$ & $92.40 \pm 0.78$ & 0.0664 \\
\hline HUVECs & 97.5 & 98.5 & 96.0 & 98.2 & 98.0 & 98.6 & $97.83 \pm 0.29$ & $98.43 \pm 0.21$ & 0.1217 \\
\hline GES-1 & 93.3 & 94.0 & 92.6 & 93.1 & 91.1 & 91.7 & $91.77 \pm 1.50$ & $93.23 \pm 0.71$ & 0.0869 \\
\hline
\end{tabular}

Survival rate of all tested samples, the mean value, and the statistical difference between the Exp (printed) and Ctrl (unprinted) groups ( $\mathrm{n}=3$ ) are presented. hADSCs, human adipose-derived stem cells; HPDLCs, human periodontal ligament cells; ARPE-19, adult retinal pigment epithelial cells; HUVECs, human umbilical vascular endothelial cells; GES-1, human gastric epithelial cells; Exp, experimental group; Ctrl, control group.
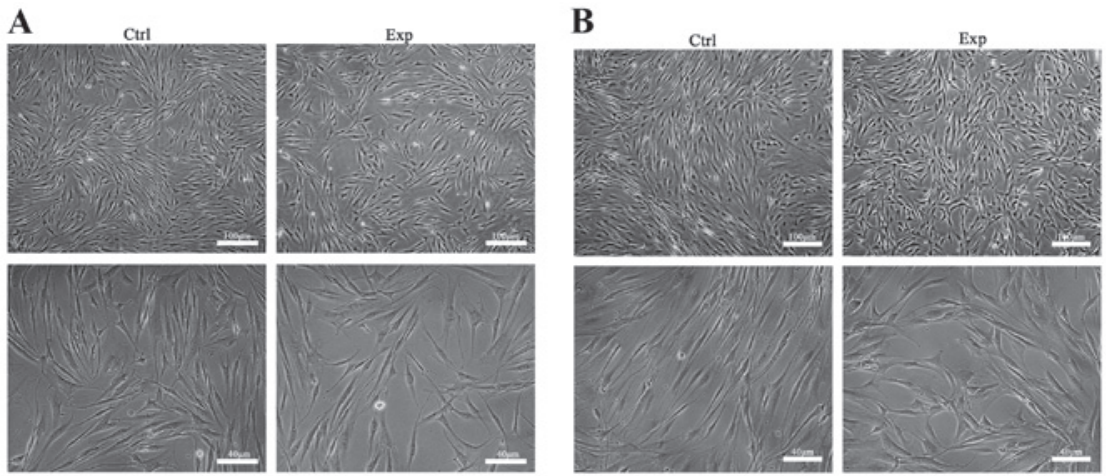
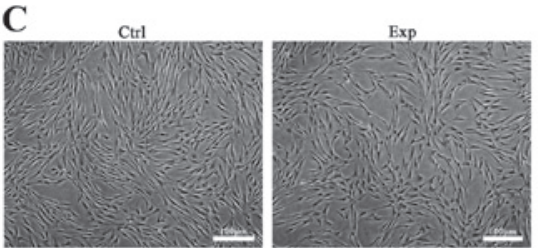

D
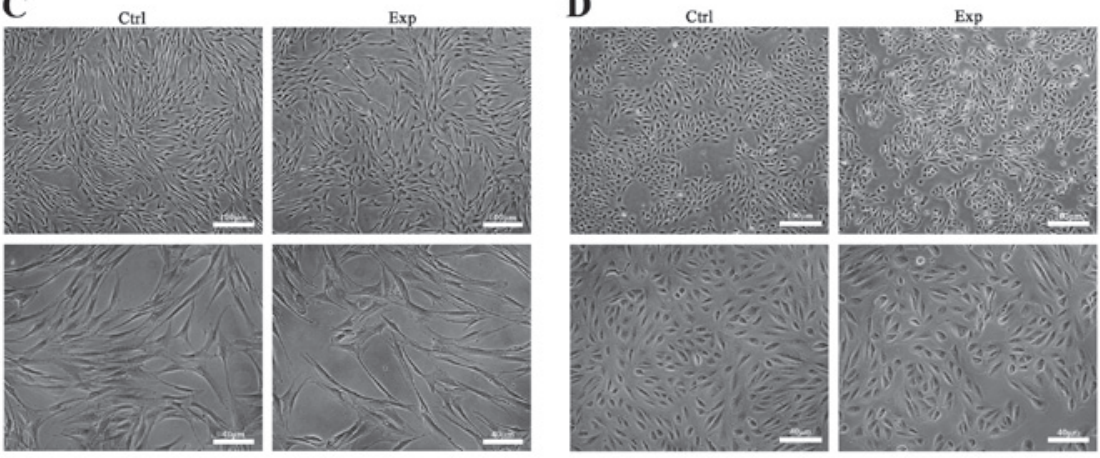

$\mathbf{E}$
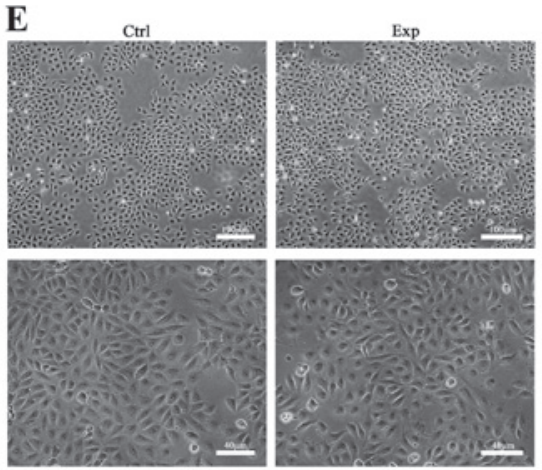
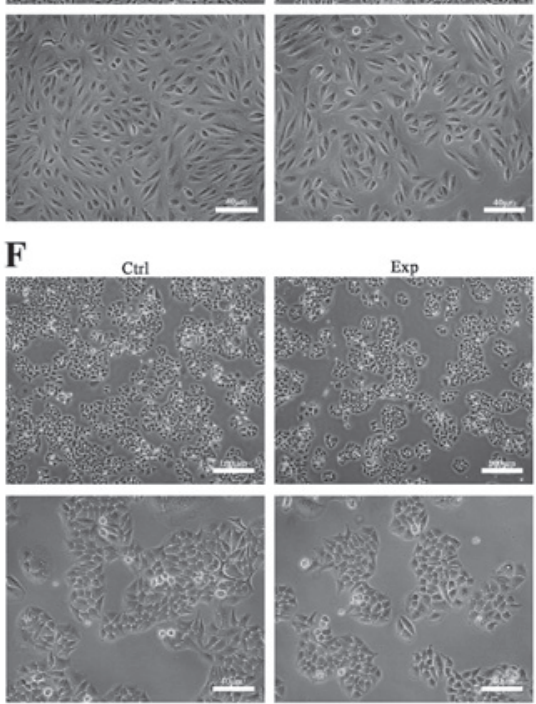
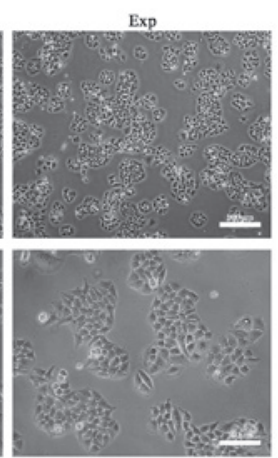

Figure 2. Cellular morphology observation under phase-contrast microscope at 5 days after electrospraying. The observed cell morphology in the in vitro cultures of (A) human fibroblasts, (B) hADSCs, (C) HPDLCs, (D) ARPE-19, (E) HUVECs and (F) GES-1 exhibited similar shape and cell density between the experimental and control groups. Images are shown at magnifications of x40 (top image) and x100 (lower image) for each group/cell type. Exp, experimental group; Ctrl, control group; hADSCs, human adipose-derived stem cells; HPDLCs, human periodontal ligament cells; ARPE-19, adult retinal pigment epithelial cells; HUVECs, human umbilical vascular endothelial cells; GES-1, human gastric epithelial cells. 

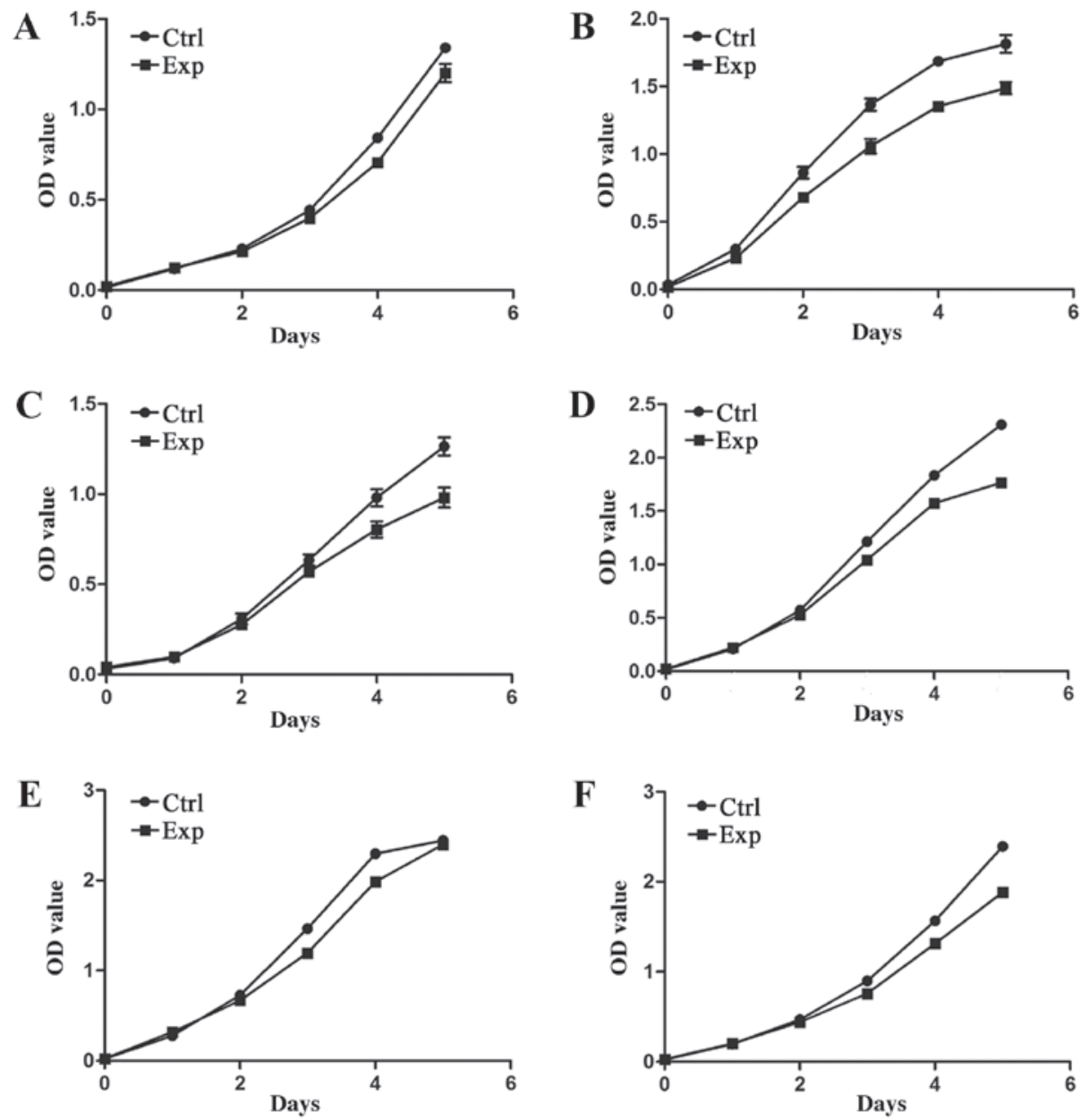

Figure 3. Cell Counting Kit-8 assay of cell proliferation viability. OD values between days 0 and 5 of incubation are shown for the post-electrospraying cell growth of the: (A) Human fibroblasts; (B) hADSCs; (C) HPDLCs; (D) ARPE-19; (E) HUVECs; and (F) GES-1. OD, optical density; Exp, experimental group; Ctrl, control group; hADSCs, human adipose-derived stem cells; HPDLCs, human periodontal ligament cells; ARPE-19, adult retinal pigment epithelial cells; HUVECs, human umbilical vascular endothelial cells; GES-1, human gastric epithelial cells.

cells were able to survive following printing, as well as to maintain their cellular morphology and proliferation capacity.

\section{Discussion}

In the present study, the cell electrospraying technology was adopted to directly print multiple living cells, in order to investigate its application as a simple and cost-effective system to produce high-throughput biomaterials. During the electrospraying process, droplets carry an electric charge that inhibits droplet accumulation. Scattered droplets, with a size within the micro- and nanoscale, are efficiently generated by changing the electric field energy (16-19). Thus, droplet movement tracks and gathering may be controlled by the movement of the nozzle, and the distance between the nozzle and the container. In addition, cell suspensions loaded into the printer are precisely placed with the assistance of CAD software, while the cell density can be controlled by the flow rate and the diameter of the nozzle $(18,20)$.

The present study indicated that multiple types of human cells can be delivered successfully using an electrospraying printer. A previous study observed that cell viability was retained subsequent to electrospraying (19). Consistent with this previous finding, the current study demonstrated that bio-electrosprayed cells have a high cell viability of $>80 \%$ (Fig. 1). In addition, the present study identified that these cells were able to proliferate (Fig. 3) and retain normal morphological characteristics (Fig. 2), indicating that damage, caused by heat and mechanical stress during printing, was not observed. Although the present study demonstrates that electrospray maintains satisfactory cell viability with high-throughput printing, driven by the appropriate voltage and flow rate, this technique continues to present challenges in offering a stable environment via a high concentration of ions $(16,20)$.

Since the ultimate goal of bioprinting is to perform in situ tissue repair with autologous cells, therapeutically treating tissue loss and organ failure with cell substitutes in vivo using extracorporeal devices remains significant (10). However, different cell types that are derived from germ layers in the human body may demonstrate a distinctly different biological performance when investigated in vitro and in vivo, particularly considering that the exogenous stimulation in environmental conditions can lead to cell phenotype shifts (21). Besides, mammalian cells strongly depend on the culture conditions and are much more sensitive to heat and mechanical stress (15). In addition, the cell numbers and types, spatial arrangement of cells, and interaction between the cells and the extracellular microenvironment in a 3D structure 
remain largely intractable, which affects the cell morphology, mechanical behavior and adhesion ability more than planar substrates $(10,22)$. Thus, building human tissue analogues in the reconstructed microenvironment remains a challenge.

Examining the feasibility of bioprinting multiple cell types may help to rebuild the human tissue and cell therapy in situ. In the present study, six types of human cells were selected to be directly printed in the same printing setting, and the cell survival and proliferation were analyzed subsequent to printing. Five of the investigated cell types demonstrated no significant difference in cell survival and proliferation when comparing the experimental and control groups, suggesting the feasibility of directly printing human cells in the printing environment used in the current study. However, differences between the experimental and control groups were observed for hADSCs in the present study, with evident differences in the mean survival rates after printing among the three samples examined (sample 1,77.7\%; sample 2,91.3\%; sample 3, 88.4\%). These differences may be due to the printing process or may result from the sample source with individual differences; therefore, the difference in cell survival in hADSCs should be studied further. In addition, three types of cells separated from human tissue and three cell lines were selected in the current study, and the cell lines demonstrated higher stability in the printing environment; therefore, differences may appear in the autologous cell printing process. Furthermore, it should be noted that only preliminary research was conducted on the cell survival ability of these multiple cell types after printing, while function tests still need to be further explored. In a further study, we will verify the feasibility of synchronous printing in a variety of human cells within bio-scaffolds with a combination of bio-electrospraying and electrospinning in a coaxial configuration. In this way, the cyclic steps of traditional tissue engineering will be reduced, including the cell seeding and incubation in the construction of scaffolds (23), while vascularized functional living tissues or organs suitable for clinical implantation will be fabricated in a shorter period of time. Bio-electrospraying has far-reaching implications and will enable significant advances in a wide range of fields, from tissue engineering to regenerative medicine.

In conclusion, the present study demonstrated the feasibility of using electrospraying technology to directly print living cells under appropriate conditions for biological and biomedical applications. The ability of this technique to organize multiple components at the appropriate time, position and amount in the well-defined 3D architecture of their native organs requires further exploration. The prospect of using the autologous cells to build bioactive models of functional tissues and organ substitutes offers a potentially revolutionary development to biomedical research and health care in the future.

\section{Acknowledgements}

Financial support was provided by the National Natural Science Foundation of China (grant no. 81372097), The Shanghai Committee of Science and Technology, China (grant nos.14441900800 and 14441900802), Project of Shanghai Jiao Tong University Medical and Engineering Cross Fund (YG2014MS06) and Shanghai Municipal Education 
Commission-Gaofeng Clinical Medicine Grant Support (20161420).

\section{References}

1. Langer R and Vacanti JP: Tissue engineering. Science 260: 920-926, 1993.

2. Ikada Y: Challenges in tissue engineering. J R Soc Interface 3: 589-601, 2006

3. Mironov V, Kasyanov V, Drake C and Markwald RR: Organ printing: Promises and challenges. Regen Med 3: 93-103, 2008.

4. Norotte C, Marga FS, Niklason LE and Forgacs G: Scaffold-free vascular tissue engineering using bioprinting. Biomaterials 30: 5910-5917, 2009

5. Boland T, Xu T, Damon B and Cui X: Application of inkjet printing to tissue engineering. Biotechnol J 1: 910-917, 2006.

6. Hutmacher DW, Sittinger M and Risbud MV: Scaffold-based tissue engineering: Rationale for computer-aided design and solid free-form fabrication systems. Trends Biotechnol 22 354-362, 2004

7. Emmert MY, Hitchcock RW and Hoerstrup SP: Cell therapy, 3D culture systems and tissue engineering for cardiac regeneration. Adv Drug Deliv Rev 69-70: 254-269, 2014.

8. Nahmias Y, Schwartz RE, Verfaillie CM and Odde DJ: Laser-guided direct writing for three-dimensional tissue engineering. Biotechnol Bioeng 92: 129-136, 2005.

9. Mironov V, Boland T, Trusk T, Forgacs G and Markwald RR: Organ printing: Computer-aided jet-based 3D tissue engineering. Trends Biotechnol 21: 157-161, 2003.

10. Miller JS: The billion cell construct: Will three-dimensional printing get us there? PLoS Biol 12: e1001882, 2014.

11. Moon S, Hasan SK, Song YS, Xu F, Keles HO, Manzur F, Mikkilineni S, Hong JW, Nagatomi J, Haeggstrom E, et al: Layer by layer three-dimensional tissue epitaxy by cell-laden hydrogel droplets. Tissue Eng Part C Methods 16: 157-166, 2010.

12. Marga F, Neagu A, Kosztin I and Forgacs G: Developmental biology and tissue engineering. Birth Defects Res C Embryo Today 81: 320-328, 2007.
13. Tasoglu S and Demirci U: Bioprinting for stem cell research. Trends Biotechnol 31: 10-19, 2013.

14. Jayasinghe SN, Eagles PA and Qureshi AN: Electric field driven jetting: An emerging approach for processing living cells. Biotechnol J 1: 86-94, 2006.

15. Xu T, Jin J, Gregory C, Hickman JJ and Boland T: Inkjet printing of viable mammalian cells. Biomaterials 26: 93-99, 2005.

16. Townsend-Nicholson A and Jayasinghe SN: Cell electrospinning: A unique biotechnique for encapsulating living organisms for generating active biological microthreads/scaffolds. Biomacromolecules 7: 3364-3369, 2006.

17. Jayasinghe SN, Warnes G and Scotton CJ: Bio-electrosprayed living composite matrix implanted into mouse models. Macromol Biosci 11: 1364-1369, 2011.

18. Jayasinghe SN, Qureshi AN and Eagles PA: Electrohydrodynamic jet processing: an advanced electric-field-driven jetting phenomenon for processing living cells. Small 2: 216-219, 2006.

19. Clarke JD and Jayasinghe SN: Bio-electrosprayed multicellular zebrafish embryos are viable and develop normally. Biomed Mater 3: 011001, 2008.

20. Fenn JB, Mann M, Meng CK, Wong SF and Whitehouse CM: Electrospray ionization for mass spectrometry of large biomolecules. Science 246: 64-71, 1989.

21. Stegemann JP and Nerem RM: Altered response of vascular smooth muscle cells to exogenous biochemical stimulation in two- and three-dimensional culture. Exp Cell Res 283: 146-155, 2003.

22. Miron-Mendoza M, Koppaka V, Zhou C and Petroll WM: Techniques for assessing 3-D cell-matrix mechanical interactions in vitro and in vivo. Exp Cell Res 319: 2470-2480, 2013.

23. Jayasinghe SN: Cell electrospinning: A novel tool for functionalising fibres, scaffolds and membranes with living cells and other advanced materials for regenerative biology and medicine. Analyst (Lond) 138: 2215-2223, 2013. 